\title{
Estudios de Cultura Maya. Una ventana a la investigación mayista internacional ${ }^{1}$

\author{
Estudios de Cultura Maya. \\ A Window on International Maya Research
}

\author{
MerCedes de la Garza \\ Investigadora emérita, Centro de Estudios Mayas, \\ Instituto de Investigaciones Filológicas, UNAM
}

Al culminar su gran labor como arqueólogo de campo, con el extraordinario hallazgo de la imponente sepultura de K'inich Janahb' Pakal en Palenque -y la reconstrucción y consolidación que configuró el estado actual en el que se encuentra la parte principal de la ciudad-Alberto Ruz Lhuillier decidió, en 1959, dedicarse al cultivo, fomento y difusión de la investigación mayista en la Universidad Nacional Autónoma de México, y se integró al entonces Instituto de Historia. En dicho instituto fundó el Seminario de Cultura Maya, como un núcleo de investigación paralelo al Seminario de Cultura Náhuatl, que dirigía el Dr. Ángel María Garibay, de quien era secretario Miguel León-Portilla.

Y asimismo, en equivalencia con la revista Estudios de Cultura Náhuatl, cuyo primer número apareció en 1959, Alberto Ruz fundó Estudios de Cultura Maya. La semejanza en el título y el formato de las dos revistas, señala Ruz, se debe al hecho de que "la Universidad se ha propuesto fomentar en forma sistemática la investigación de dos de las grandes culturas autóctonas que constituyen la raíz y gloria de la Nación Mexicana."” A nadie mejor pudo recibir la Universidad Nacional para esta importante labor que al arqueólogo más riguroso y destacado en la investigación mayista que había en ese momento en México.

Las dos revistas se proponían:

[...] reunir estudios de muchos especialistas, o de muchos estudiantes que se avezan a serlo, y ponerlos a disposición de los estudiosos - que debieran serlo todos los mexicanos, que debieran serlo todos los hombres en capacidad de ello- para que ellos encuentren, o base de nueva edificación, o piedra de escándalo para destruir, con razones, lo que se propuso acaso sin ellas. ${ }^{3}$

A Estudios de Cultura Maya, Ángel María Garibay la llamó varias veces "un gemelo de este modo de estudios". ${ }^{4}$ Garibay expresa su deseo de que se mantuvieran

1 Una parte de este trabajo deriva de mi artículo "Estudios de Cultura Maya. Un diálogo entre mayistas a lo largo de 40 años”, Historia Mexicana, vol. L, Núm. 4, pp. 719-729, México, El Colegio de México, 2001.

2 "Presentación" a Estudios de Cultura Maya, vol. I, México, Universidad Nacional Autónoma de México, Facultad de Filosofía y Letras, Seminario de Cultura Maya, 1961.

3 Ángel María Garibay, "Proemio", en Estudios de Cultura Náhuatl, I, 1959, p. 7.

${ }^{4}$ En Alberto Ruz Lhuillier, “Ángel María Garibay Quintana (1892-1967)”, en Estudios de Cultura Maya, VII, 1968, pp. 394-395. 
las condiciones externas para la consolidación de la revista sobre los nahuas, deseo que se cumplió, y con creces, ya que Estudios de Cultura Náhuatl, gracias a la dedicación constante que desde entonces hasta hoy le brindó el muy distinguido nahuatlato Miguel León-Portilla y al Instituto de Investigaciones Históricas, ha llegado ya a su volumen 50 .

En cuanto a Estudios de Cultura Maya, su destino era tomar otro camino. La revista nunca fue en realidad una publicación del Instituto de Historia, pues antes de la edición del primer volumen, el Seminario de Cultura Maya se separó del instituto, y a mediados de 1960 fue adscrito a la Facultad de Filosofía y Letras. Este seminario, en su vertiente docente y de iniciación a la investigación, continúa hoy adscrito a la misma facultad y a los Institutos de Investigaciones Históricas y Filológicas, formando parte de los programas de posgrado en Historia y en Estudios Mesoamericanos. Su titular siguió siendo Alberto Ruz, hasta 1977. Desde esa fecha hasta 2009 lo impartió Mercedes de la Garza.

Pero en sus orígenes, este seminario, pensado como un centro de investigación más que como un curso, se propuso, en su creación:

La investigación de campo y de gabinete, la docencia, la publicación de obras tanto de carácter especializado como de amplia divulgación en nivel universitario, reedición de fuentes históricas, conferencias y discusiones de mesa redonda; en fin, las diferentes formas de contribuir a la adquisición de nuevos conocimientos sobre la vida y la cultura de los mayas [antiguos y presentes] y de darlos a conocer. ${ }^{5}$

Sin embargo, su principal labor durante algunos años, fue la edición de la revista, en la que colaboraron desde el principio, con una ejemplar solidaridad científica, los más connotados mayistas del mundo en ese momento, como Eric Thompson, Herbert Spinden, Alfred Kidder, George Kubler y Gordon Willey. Y como investigadores permanentes del seminario fueron nombrados Calixta Guiteras Holmes, Alfonso Villa Rojas, Alfredo Barrera Vásquez y César Lizardi Ramos. El interés de los mayistas por Estudios de Cultura Maya se debió también a que, en ese momento, la revista era la única publicación periódica especializada en los mayas en el ámbito internacional, por lo que con gran entusiasmo enviaron sus colaboraciones, que contribuyeron a crear el sólido prestigio del que goza hasta ahora la revista.

El primer número se editó en 1961, y los siguientes, hasta el volumen VII, aparecieron anualmente, gracias al rigor y la dedicación de Alberto Ruz. El volumen VIII se publicó en 1970, fecha en la cual el seminario se convirtió en un Centro de Estudios Mayas, con aproximadamente 20 investigadores de varias disciplinas, y se empezaron a cumplir los propósitos iniciales del seminario. Tres años después, el centro pasó a formar parte del nuevo Instituto de Investigaciones Filológicas, fundado por Rubén Bonifaz Nuño. En el nuevo centro aumentaron las colabora-

\footnotetext{
${ }^{5}$ Alberto Ruz Lhuillier, “Prólogo”, en Estudios de Cultura Maya, I, 1961.
} 
ciones de sus miembros para la revista, aunándose a su carácter internacional, y Estudios de Cultura Maya empezó a dar a conocer las líneas de investigación y los trabajos de ese núcleo de mayistas.

Debido a las crecientes actividades del Centro de Estudios Mayas, para la preparación del volumen X se creó una comisión editorial, formada por Carlos Serrano (antropólogo físico), Lorenzo Ochoa (arqueólogo) y Mercedes de la Garza (historiadora), quienes se hicieron cargo, tanto de Estudios de Cultura Maya como de las otras publicaciones que se habían fundado en el centro: "Serie Cuadernos" y "Publicaciones Especiales".

A principios del mismo año de la aparición del volumen X (1977), un significativo hecho se produjo en el Centro de Estudios Mayas: su fundador y director, Alberto Ruz Lhuillier, se fue de la Universidad Nacional para desempeñar el cargo de director del Museo Nacional de Antropología, puesto que ocuparía hasta su muerte, ocurrida en 1979. En el CEM, fue nombrada directora Mercedes de la Garza, quien a la vez dirigió la revista, a la que mantuvo como publicación prioritaria, al lado de la Comisión Editorial, durante trece años (de 1977 a 1990), a pesar de la creación de nuevas publicaciones, como la "Serie de Fuentes para el Estudio de la Cultura Maya” (1980).

Hasta 1992, Estudios de Cultura Maya mantuvo un buen ritmo temporal, ya que se editaron nueve números (XI, 1978; XII, 1979; XIII, 1981; XIV, 1982; XV, 1984; XVI, 1985; XVII, 1988; XVIII, 1991 y XIX, 1992), a pesar de que los investigadores han tenido, como parte de sus obligaciones para con el centro, la dura tarea de preparar los Congresos Internacionales de Mayistas, creados en 1985 y realizados cada dos años al principio, y cada tres a partir de 1989.

Infortunadamente, de 1992 a 2000 sólo apareció el volumen XX (en 1999), lo que causó desaliento en los colaboradores, sobre todo en algunos mayistas de otros países que antes enviaban con regularidad trabajos para darlos a conocer a través de Estudios de Cultura Maya. Ya no fue posible que el coordinador del centro fuera también director de la revista a causa de sus crecientes y cada vez más complejas responsabilidades. De este modo, desde la publicación del volumen XXI, la revista fue encomendada a algunos investigadores, como Ana Luisa Izquierdo, editora de ese volumen. En él ya no aparece mención de la Comisión Editorial del Centro, sino sólo del Consejo Editorial, constituido por destacados investigadores mayistas nacionales y extranjeros. En el volumen XXIII (2003), editado por Mario Humberto Ruz, coordinador entonces del centro, y Martha Ilia Nájera, por decisión de los editores, la comisión del CEM se denominó Comité Editorial y no aparece el Consejo Editorial. El volumen XXIV también estuvo a cargo de Ruz y Nájera. En el XXV (2005), el editor fue sólo Mario Humberto Ruz, y a partir del XXVI, fungieron como editores Ruz y Claudia M. Báez, hasta que fue nombrada directora de la revista Maricela Ayala (vol. XXX, 2007), y editores, Báez, Roberto Romero y Mauricio Ruiz Velasco. Y desde el volumen XLIV dirigió la revista Mario Humberto Ruz, fungiendo como editora Claudia M. Báez y como editor de la revista digital Roberto Romero. 
Los cambios en los comités y el consejo se debieron a la necesidad de adecuar la revista a los requerimientos de los índices nacionales e internacionales de revistas científicas; así, a través del tiempo se han ido regularizando las características editoriales, pero sin mengua de la calidad académica de las contribuciones.

En cuanto a su formato, la revista se inicia con una nómina de los miembros del Centro de Estudios Mayas — dicha lista, a partir del volumen XLVIII (2016) se consigna al final de la publicación-y los nombres del fundador: Alberto Ruz Lhuillier, del director, los editores y el Consejo Editorial. En seguida, aparecen el índice, los artículos, reseñas, necrológicas y notas diversas, y al final, breves curricula de los colaboradores en el número en cuestión. En los primeros números, se incluía también una presentación del volumen por parte del coordinador del centro. Los artículos se agrupan por disciplinas y al final había una lista de las publicaciones del centro, que hoy se ha limitado a las publicaciones recientes destacadas en las solapas; se han añadido las normas editoriales de la revista. Cada uno de los artículos lleva un abstract en español y en inglés.

Hasta 1999 (Vol. XX) cada número de esta publicación era un grueso volumen de alrededor de quince artículos, acompañados de mapas, cuadros e ilustraciones a tinta o fotografías, y cerca de 500 páginas, por lo que era imposible editar un número por semestre, y muy difícil hasta por año. La carátula llevaba en el centro un dibujo a línea de un relieve de la ciudad de Palenque, Chiapas, conocido como "El Escriba".

Pero la normatividad de los índices de revistas, particularmente el de CONACYT, han obligado a la periodicidad semestral, que se logró también gracias a Mario Humberto Ruz, en 2003, quien fungía en ese momento como coordinador del centro y coeditor de la revista, y desde entonces se publican dos números anuales. Esto ha dado mucha agilidad a Estudios de Cultura Maya, y la oportunidad de conocer en un tiempo razonable las nuevas investigaciones. Y, además, permitió que en 2010 la revista fuera aprobada para ser integrada al "Índice de Revistas Mexicanas de Investigación Científica y Tecnológica de CONACYT". En 2015 ella fue de nuevo evaluada para permanecer en el que desde 2016 se denomina "Sistema de Clasificación de Revistas Mexicanas de Ciencia y Tecnología". Además, hoy día Estudios ha sido incluida en índices internacionales, como Scopus, Latindex, DOAJ, Scielo o Redalyc, colocándose en niveles de excelencia tanto nacional como internacional. Gracias a todo ello, actuando en primer lugar su calidad académica, Estudios de Cultura Maya se ha posicionado en el primer lugar entre todas las revistas mexicanas de Artes y Humanidades. ${ }^{6}$

A partir del volumen XXII (2002), también bajo la coordinación del centro, a cargo de Mario Humberto Ruz, cambió el diseño de la portada: con distintos motivos al centro o en un solo color, y una banda vertical al lado izquierdo, en lo alto de la cual se conserva el dibujo a línea de El Escriba, que identifica a la publicación desde sus orígenes.

\footnotetext{
${ }^{6}$ Agradezco los datos que me proporcionaron Lynneth Lowe y Claudia Báez.
} 
La directora actual de la revista es Lynneth Lowe. A partir del volumen XLVIII, de 2016, asumiendo la responsabilidad de la calidad académica de los artículos, el Comité Editorial de la revista está conformado por un 60\% de miembros externos y un 40\% de académicos de distintas entidades académicas de la UNAM, con representación de las áreas disciplinarias incluidas en la publicación: arqueología, epigrafía, iconografía, historia, linguiística, etnohistoria, antropología social y áreas nuevas, como música y literatura. Y el Consejo Editorial se conforma con destacados académicos en el ámbito de la investigación mayista: en el 92\% miembros externos a nuestra institución, y un $77 \%$ de miembros adscritos a instituciones académicas internacionales (Guatemala, Estados Unidos, España, Francia, Italia y Alemania).

Estudios de Cultura Maya siempre ha constituido un medio de comunicación entre mayistas del mundo entero y de divulgación de los distintos aspectos y enfoques que en diversos países se da a la investigación sobre los mayas. Así, está destinada principalmente a investigadores de la cultura maya, pero también de las culturas indígenas mesoamericanas, del norte de México y antiguas en general. Edita trabajos en español, inglés, francés y lenguas mayances; los artículos en otros idiomas, como alemán, ruso o japonés se traducen al español. Incluye algunas reseñas y notas necrológicas, como hemos mencionado, pero el peso fundamental está en los artículos científicos, pues no es una revista de difusión. La distribución ha estado a cargo de la Dirección General de Publicaciones de la UNAM, y hasta el volumen XVIII un buen número de ejemplares se enviaba por intercambio a bibliotecas de instituciones dedicadas a la investigación en México y en otras partes del mundo, así como a los colaboradores y a mayistas destacados nacionales y extranjeros. El directorio de especialistas incluía investigadores de diversas disciplinas en México, Estados Unidos, Canadá, Guatemala, El Salvador, Honduras, Belice, España, Alemania, Francia, Italia, Japón, Rusia y Australia, entre otros países. Actualmente hay nuevas políticas de distribución de la revista para su venta, y el tiraje ha disminuido de 2000 a 300 ejemplares.

Pero en cuanto a su contenido, Estudios de Cultura Maya ha conservado a lo largo de estos 56 años la calidad de excelencia con la que se creó, pues sigue contando con la colaboración de mayistas distinguidos en distintas disciplinas y los trabajos siempre son sometidos a dictámenes rigurosos.

La revista, como su nombre lo expresa, no se centra en una disciplina, sino en una cultura, por lo que su carácter es multidisciplinario, como el del Centro de Estudios Mayas. Recibe trabajos realizados con los diversos enfoques científicos que se dan a la investigación sobre el pueblo maya, su historia y sus creaciones, y no se limita a los mayas prehispánicos, sino que incluye la historia del área maya en la época colonial y los grupos mayances actuales. De este modo, Estudios de Cultura Maya publica trabajos arqueológicos, históricos, epigráficos, estéticos, linguiísticos, etnológicos, de antropología física, matemáticos, arqueoastronómicos, sociológicos, etc., o bien, artículos en los que se integran dos o más disciplinas, y está abierta a cualquier tipo de investigación que se ocupe del universo maya y que esté realizada con rigor científico. 


\section{Sobre el contenido de Estudios de Cultura Maya ${ }^{7}$}

Estudios ha sido un importante medio para dar a conocer trabajos e informes arqueológicos, los cuales muchas veces (sobre todo en México) se quedan archivados como reportes de trabajo de campo, sin llegar al conocimiento de los otros investigadores. Se han publicado desde complejos artículos, resultado de la investigación arqueológica, hasta los informes preliminares y avances de un proyecto. Entre los muchos trabajos que de esta disciplina ha editado Estudios de Cultura Maya podemos mencionar "Algunas consideraciones sobre las pinturas de Mul-Chic, Yucatán” de Román Piña Chan (IV); "Brainerd y Ruppert en Xpuhil en 1949” de Harry E. D. Pollock (VI); “Cerámica de la región de Palenque, México” de Robert Rands (VI); "Ceramic Wares in the Maya Area: A Clarification of an Aspect of the Type-Variety System and Presentation of a Formal Model for Comparative Use" de Jeremy Sabloff y Robert E. Smith (VIII); "Microscopic Analysis of Chipped Stone Tools from Barton Ramie, British Honduras" de Richard Wilk (X); "Puertos costeros del Postclásico Temprano en el norte de Yucatán” de Anthony P. Andrews (XI); "Los asentamientos prehispánicos y la arquitectura en la isla Can Cun, Quintana Roo" de Ernesto Vargas Pacheco (XI); "Hilos del mismo tejido cultural: el área maya y la Costa del Golfo" de Tomás Pérez Suárez (XXIII); “Edzná y la región occidente del Puuc" de Antonio Benavides C. (XXIII); "Secuencia constructiva de las plazas en el grupo principal de El Palmar, Campeche, México" de Kenichiro Tsukamoto, Hirokazu Kotegawa y Luz Evelia Campaña (XXXIX); "Cronología cerámica en Cancuén: historia de una ciudad del Clásico Tardío” de Melanie Forné, Silvia Alvarado y Paola Torres (XXXVIII); "Politics in the Western Maya Region (I)" de Péter Bíró (XXXVIII); "Las antiguas colecciones arqueológicas de Yucatán en el Museo Americano de Historia Natural” de Adam T. Sellen y Lynneth S. Lowe (XXXIII); "La legitimación de la realeza entre los mayas del Preclásico Tardío. Los mascarones de El Tigre, Campeche” de Ernesto Vargas Pacheco (XXXVI); "Las bases económicas de una entidad política maya. El caso de Toniná” de Eric Taladoire (XLVIII).

Y se han incluido nuevas líneas de investigación, como "Arqueoacústica maya. La necesidad del estudio sistemático de efectos acústicos en sitios arqueológicos" de Clara Garza, Andrés Medina, Pablo Padilla, Alejandro Ramos y Francisca Zalaquet" (XXXII). Sobre arqueoastronomía se han publicado "Astronomía sin telescopios. Conceptos mayas del orden astronómico” de John R. Sosa (XV); "Identification of Postclassic Maya Constellations from the Venus Pages of the Dresden Codex" de Changbom Park y Heajoo Chung (XXXV); "Astronomía en la arquitectura maya de Chichén Itzá: una reevaluación” de Ivan Šprajc y Pedro Francisco Sánchez Nava (XLI).

\footnotetext{
${ }^{7}$ Los trabajos y autores mencionados de aquí en adelante se han seleccionado aleatoriamente, con el único criterio de mostrar la variedad y la riqueza del contenido de Estudios de Cultura Maya. El número de la revista se indica entre paréntesis.
} 
Y entre los informes y avances de proyectos se pueden destacar el de los trabajos en Toniná de 1979, realizados por Pierre Becquelin y Eric Taladoire, y el de Nuevo Jalisco, Chiapas, por Ramón Carrasco (volumen XIII). Además, cito los siguientes: "Preliminary Report of the Conference on the Prehistoric Ceramics of the Maya Lowlands" por Patrick Culbert (VI); "Kukulcán y un culto fálico en Chichén Itzá, Yucatán, México" (VIII) por William Folan; "Notas preliminares sobre el proyecto Arqueología de las Tierras bajas noroccidentales del área maya" por Lorenzo Ochoa (X); "Trabajos recientes en Xcaret, Quintana Roo, por María José Con (XVIII); "Excavations in Nakum Structure 99: New Data on Protoclassic Rituals and Precolumbian Maya Beekeeping" escrito por Jaroslaw Zralka, Wieslaw Koskul, Katarzyna Rodnicka, Laura Elena Sotelo Santos y Bernard Hermes (XLIV); "De la autonomía política y cultural de la provincia de Rio Bec" de Phillipe Nondédéo, Julie Patrois, Alfonso Lacadena, M. Charlotte Arnauld, Eric Taladoire y Dominique Michlet (XXXVI) y "Under the Rule of the Snake Kings: Uxul in the $7^{\text {th }}$ and $8^{\text {th }}$ Century" de Nikolai Grube, Kai Delvendahl, Nicolaus Seefeld y Beniamino Volta (XL).

Otros reconocidos arqueólogos que han colaborado en la revista son Gordon Willey (I y V), Peter J. Schmidt (X), Gareth W. Lowe (II y V), Carlos Navarrete (VIII y XVI), John E. Clark (XIII), Andrés Ciudad Ruiz (XIII), Carlos Álvarez (XIV), David Webster (volumen XIV); Roberto García Moll (XII); John E. Clark y Thomas A Lee Jr. (XII); Antonio Benavides (XVIII y XXX); Anabel Ford (XVIII); Edgar Carpio Rezzio (XXI); Stanislaw Iwaniszewki (XXVIII); Eric Taladoire (XLVIII); Sylviane Boucher (XXXIX) y Vera Tiesler (XXXVIII).

Asimismo, hay múltiples artículos sobre historia y religión maya, tanto de la época prehispánica como de los mayas coloniales y actuales. Cabe mencionar los trabajos de Calixta Guiteras Holmes, "La magia en la crisis del embarazo y parto en los actuales grupos mayances de Chiapas" (I); Lothar Knauth, "El juego de pelota y el rito de la decapitación" (I); Robert Laughlin, "El símbolo de la flor en la religión de Zinacantán" (II); Alfonso Villa Rojas, "El nagualismo como recurso de control social entre los grupos mayances de Chiapas, México" (III), y William R. Holland, "Psicoterapia maya en los altos de Chiapas" (III), que formaron parte del grupo inicial de colaboradores de la revista. Cabe mencionar, además, los trabajos de Robert Bruce, "The Popol Vuh and the Book of Chan K'in" (X); María Montoliú, "Algunos aspectos del venado en la religión de los mayas de Yucatán" (X); Eric Thompson, "Maya Creation Myths" (partes 1-2, volúmenes V y VI); Eva Alejandra Uchmany, "Supervivencias de formas religiosas prehispánicas en la región maya" (III); William Folan, "Kukulcán y un culto fálico en Chichén Itzá, Yucatán, México" (VIII); Miguel León-Portilla, "Nuevas aportaciones al tema del tiempo entre los mayas" (XVII); de Laura Sotelo y Carmen Valverde, "Los señores de Yaxchilán. Un ejemplo de la felinización de los gobernantes mayas" (XIX); "Discursos ceremoniales tzotziles: una joya poco estudiada" de Ulrich Köhler (XXXIII); "The Gods of Yucatán from A.D. 1560 to 1980" de Bruce Love (XXXVII); Martha Ilia Nájera, "El mono y el cacao: la búsqueda de un mito a través de los relieves del Grupo de la Serie Inicial de Chichén Itzá (XXXIX); "El pasado y el presente: las moradas 
de 'los antiguos' en la cosmología maya peninsular actual” de David de Ángel García (XLIII).

Los artículos epigráficos abundan en Estudios de Cultura Maya, sobre todo en los primeros volúmenes, debido a que el Centro de Estudios Mayas se formó cuando se integraron el Seminario de Cultura Maya y el Seminario para el Estudio de la Escritura Maya, fundado también por Alberto Ruz Lhuillier, que había estado adscrito a la Coordinación de Humanidades, bajo la dirección de Daniel Cazés, y al que pertenecieron Leonardo Manrique y Maricela Ayala, entre otros. Este seminario se proponía el estudio sistemático de la escritura maya prehispánica, y organizó en 1966 el Primer Seminario Internacional para el Estudio de la Escritura Maya, al que asistieron los más reconocidos epigrafistas de ese entonces, procedentes de Alemania, Rusia y Estados Unidos, principalmente; el volumen VII de la revista se dedicó íntegramente a la publicación de los trabajos presentados en esa reunión académica. En 1963-1964 se publicaron en Estudios de Cultura Maya "Historical Data in the Inscriptions of Yaxchilan" (partes I-II, vols. III y IV), trabajos iniciales de la destacada estadounidense Tatiana Proskouriakoff, de gran trascendencia para la epigrafía y para la investigación mayista en general, de los que parte, al lado de los trabajos de Heinrich Berlin, la línea de interpretación histórica de las inscripciones mayas, que ha cobrado especial importancia en nuestros días. Otros epigrafistas que han colaborado en la revista son David Kelley, "Fonetismo en la escritura maya" (II); Yuri Knorozov, "Aplicación de las matemáticas al estudio linguí́stico" (III); "Principios para descifrar los escritos mayas" (V) e "Investigación formal de los textos jeroglíficos mayas" (VII); Thomas Barthel, "Comentarios a las inscripciones clásicas tardías de Chichén Itzá" (IV) y otros trabajos en los volúmenes V, VI y VII; Dieter Dütting, "Algunas consideraciones sobre el trabajo de H. Berlin 'The Palenque Triad”' (V) y una colaboración más en el volumen VII; Floyd G. Lounsbury y Michael D. Coe, "Linguistic and ethnographic data pertinent to the "cage' glyph of Dresden 36c" (VII); Maricela Ayala, "Relaciones entre textos y dibujos en el Códice de Dresde" (VII); Roberto Escalante, "Método de descifre" (VII); Mary Ellen Miller y David S. Stuart, "Dumbarton Oaks Relief Panel 4" (XIII); Carolyn Tate, "Summer Solstice Ceremonies Performed by Bird Jaguar III of Yaxchilan, Chiapas (XVI); Nicholas A. Hopkins, "Classic Mayan Kinship Systems: Epigraphic and Ethnographic Evidence for Patrilineality" (XVII); Michel Davoust, "Nouveaux Commentaires des Textes de Quelques Vases Polychromes de la periode Classique Maya” (XVIII); "Las orejeras de K'inich Janahb' Pakal: comentarios sobre una inscripción olvidada de Palenque" de Guillermo Bernal Romero (XXXI); "Los señores de la entidad política de "Ik" de Erik Velásquez García (XXXIV); "Los dioses, el gobernante y la comunidad. Las estrategias político-religiosas del Gobernante 2 de Dos Pilas, Guatemala" de María Elena Vega Villalobos (XL), y "Acerca del jeroglífico [T544.501] y las colectividades geopolíticas entre los mayas clásicos", de Rogelio Valencia Rivera y Alejandro Sheseña Hernández (XLVIII).

De historia del arte e iconografía citaremos los trabajos de George Kubler, "Chichén Itzá y Tula" (I) y "Pintura mural precolombina" (VI); el primero de estos 
artículos (que va mucho más allá de un texto sobre arte) despertó una histórica polémica con Alberto Ruz, quien en el volumen II escribió "Chichén Itzá y Tula, comentarios a un ensayo” (II), al cual Kubler contestó, en el mismo volumen II, "Réplica del Doctor Kubler al trabajo de Alberto Ruz que antecede"; de Martha Foncerrada de Molina se editó "La arquitectura Puuc dentro de los estilos de Yucatán" (II), y otros autores en estas disciplinas han sido Juan Antonio Valdés, con "Los mascarones del grupo 6C-XVI de Tikal: análisis iconográfico para el Clásico Temprano" (XVIII); Clemency Coggins, "The Manikin Scepter: Emblem of Lineage” (XVII); Michel Graulich, "Oblique Views and Three-Dimensionality in Maya Art" (XVIII); "Símbolos de poder: un análisis comparativo entre la iconografía del Clásico Maya y los códices mixtecos" de Manuel A. Hermann Lejarazu (XXX); "Análisis iconográfico preliminar de fragmentos de las vasijas estilo códice procedentes de Calakmul" de Ana García Barrios (XXXVII); "Guacamaya: símbolo de temporalidad y fertilidad en dos ejemplos de pintura mural” de Lourdes Navarijo Ornelas (XXXIX); "El laberinto literario de las poetas mayas yucatecas contemporáneas" de Óscar Ortega Arango (XLII); "World Renewal Rituals among the Postclassic Yucatec Maya and Contemporary Ch'orti’ Maya” de Gabrielle Vail y Matthew G. Looper (XLV).

Se han editado diversos estudios sobre la época colonial, que dan cuenta de este momento histórico tan importante en el destino de los grupos mayances que perviven hasta hoy, como los realizados por Alfredo Barrera Vásquez, "Contrata de un maya de Yucatán, escrita en su lengua materna, para servir en Cuba, en 1849" (I); Ian Graham, "Juan Galindo, Enthusiast” (III); Luis Millet Cámara, "La encomienda de Sor Águeda del Padre Eterno y Sor Feliciana de San Antonio en Chiapas" (XIII); Robert Carmack, "New Quichean Chronicles from Highland Guatemala" (XIII); Ana Luisa Izquierdo, "Documentos de la división del Beneficio de Yaxcabá. El castigo de una idolatría” (XVII); Dolores Aramoni, "Los indios constructores de Palenque en un documento del siglo XVIII" (XVIII); Mario Humberto Ruz, "El conquistador y el jurisconsulto. Testimonios sobre el Itzá" (XIX); "Encontrarán su comida entre los árboles [...] entre las rocas" (Libro de Chilam Balam de Chumayel). Épocas de padecimiento y dispersión para los mayas de Yucatán" por Ruth Gubler (XIX); de Gudrun Lohmeyer, "La fundación del convento de Comitán” (XIX); de Lorraine Williams-Beck y Eduardo López de la Rosa, "Historia de tres ciudades: Ah Kin Pech, Acanmul y San Francisco de Campeche” (XX); "La Theologia Indorum de Vico en lengua quiché" de René Acuña (XXIV); "El Popol Vuh: algunas consideraciones históricas" de Gudrun Lenkersdorf (XXIV); "It is their drinking that hinders them': balché and the use of ritual intoxicants among the colonial Yucatec Maya” de John F. Chuchiak IV (XXIV); "Reducción de indios infieles en la Montaña del Chol: la expedición del Sargento Mayor Miguel Rodríguez Camilo en 1699", de Francisco Luis Jiménez Abollado (XXXV); "Nuevas luces sobre un antiguo testimonio acerca de los mayas: el informe de la expedición comandada por Juan de Grijalva” de María del Carmen León Cázares (XLV).

Diversos artículos etnográficos, etnológicos y de antropología social se han dado a conocer en la revista; entre ellos se cuentan los de reconocidos especia- 
listas en el ámbito internacional como Evon Z. Vogt, "Some Aspects of Zinacantan Settlement Patterns and Ceremonial Organization" (I); Alfonso Villa Rojas, "Distribución y estado cultural de los grupos mayances del México actual" (II); Carlo Antonio Castro, "Una relación tzeltal del carnaval de Oxchuc" (II); Roberta Montagu y Eva Hunt, "Nombre, autoridad y el sistema de creencias en los Altos de Chiapas" (II); Henri Favre, "Notas sobre el homicidio entre los chamulas" (IV); Frank A. Cancian, "Efectos de los programas económicos del Gobierno Mexicano en las Tierras Altas mayas de Zinacantán” (V); Rubén E. Reina, “Town, Community and Multicommunity; The Theoretical Implications of a Guatemala Case” (V); June C. Nash, "Época para cazar brujos" (IX), y otros artículos en los volúmenes IV y VIII; de Gary H. Gossen, "Cuatro mundos del hombre: tiempo e historia entre los chamulas" (volumen XII); de Antonio García de León, "Algunas consideraciones sobre los choles" (XII); "El carnaval de Bachajón" de Alain Breton y Aurore Becquelin-Monod (volumen XII); de Perla Petrich, "Los mochós cuentan de dónde vino el fuego" (XV); de John B. Haviland, "Lenguaje ritual sin ritual” (XX); de José Alejos, "Vencer o morir. Mitología y sociedad entre los choles" (XX); "La memoria de los ancianos mayas prehispánicos. Historiografía desde una perspectiva de género (siglos XX y XXI) (XXXII)”, de Rocío García Valgañón; “Los dos cuerpos mayas. Esbozo de una antropología elemental indígena” de Pedro Pitarch (XXXVII); "Memorar la cultura: modos de mantener y formar las identidades mayas modernas" de Felix A. Kupprat (XXXVIII).

Entre las colaboraciones en linguiística destacan las de Pedro Carrasco, "Los nombres de persona en la Guatemala antigua" (IV); Otto Schumann, "Los reverenciales en las lenguas mayas" (XVI); "Introducción al sistema verbal del chortí de Guatemala” de Raúl del Moral (XVII); Barbara Pfeiler, "El uso de dos lenguas en contacto" (XVII); Carlos Lenkersdorf, "Del género y la perspectiva tojolabal” (XX); "Cinco textos oraculares mayas" de René Acuña (XX); "Paralelismo, ciclicidad y creatividad en el arte verbal maya yucateco" de Valentina Vapnarsky (XXXII); "Descripción fonética de los tonos del maya yucateco" de Martín Sobrino Gómez, (XLI).

Pocos son los trabajos de antropología física que publicó Estudios de Cultura Maya, mientras existió en el Centro esa especialidad. Se citan aquí los de Carlos Serrano, "Estudio comparativo de los dermatoglifos digitales de los zoques y otros grupos indígenas del sureste de México" (X); "The Biological Affinity of the Ancient Populations of Altar de Sacrificios and Seibal" de Donald M. Austin; Julieta Aréchiga, "Antropometría nutricional en la población infantil de Chan Kom, Yucatán” (XI); Lourdes Márquez Morfín, “La dieta maya prehispánica de la costa yucateca” (XVIII); "Afinidades biológicas de la población de Oxtankah en el ámbito regional de la Península de Yucatán" de Andrea Cucina y Allan Ortega Muñoz (XLIV); "Manejo postsacrificial del cuerpo humano: evidencias e implicaciones rituales en un entierro del Clásico Terminal en Lagartero, Chiapas" de Judith L. Ruiz González, Carlos Serrano Sánchez y Sonia Rivero Torres (XLVIII).

En el panorama general que aquí he presentado se puede constatar que la publicación periodica del Centro de Estudios Mayas del Instituto de Investigaciones 
Filológicas de la UNAM, no sólo ha logrado permanecer a lo largo de 56 años, sino que ha alcanzado una fuerte consolidación como una revista reconocida nacional e internacionalmente, en la que los mayistas más distinguidos del siglo XX y lo que va del XXI, así como muchos otros, entre los cuales se cuentan jóvenes investigadores mexicanos, centroamericanos, norteamericanos, japoneses, coreanos y europeos, han podido dar a conocer sus trabajos recientes, fomentando el diálogo científico sobre el pueblo maya y su cultura en el ámbito internacional. Actualmente, la revista ocupa el primer lugar entre todas las revistas mexicanas de Artes y Humanidades.

Por mi parte, como investigadora y responsable de Estudios de Cultura Maya durante 14 años, agradezco muy profundamente el gran esfuerzo y entrega, no sólo de los coordinadores, los editores y los directores de la revista, sino de todos los investigadores del Centro de Estudios Mayas, y asimismo, de la Dirección y el Departamentos de Publicaciones del Instituto de Investigaciones Filológicas, por haber mantenido y enriquecido aquel inicial reto editorial e impulso creativo de Alberto Ruz Lhuillier, que ha desarrollado una trayectoria de excelencia entre las publicaciones especializadas del mundo. 
